Ilya Kister, MD

Geoffrey Kuesters, PhD

Eric Chamot, MD, PhD

Mirza Omari, MPH

Kim Dontas, RN

Mary Yarussi, RN

Meena Subramanyam,

$\mathrm{PhD}$

Joseph Herbert, MD

Correspondence to

Dr. Kister:

ilya.kister@gmail.com

\title{
IV immunoglobulin confounds JC virus antibody serostatus determination
}

OPEN

ABSTRACT

Objective: To determine the impact of therapeutic infusion of IV immunoglobulin (IVIg) on John Cunningham virus antibody (JCV Ab) serostatus and level in serum.

Methods: We carried out a retrospective analysis of serum levels of JCV Ab among STRATIFY-2 trial enrollees from 2 multiple sclerosis centers who were exposed to IVIg during the trial. For the subset of eligible patients, we estimated mean linear trends while on IVIg and after stopping IVIg with a linear mixed-effects model.

Results: The JCV Ab seropositivity rate in the group of patients that was recently exposed to IVIg was $100 \%$, which is significantly higher than in the IVIg-naive population ( $58 \%, p<0.001)$. The seropositivity rate in the patient group with remote IVIg exposure was similar to that in the IVIgnaive population ( $67 \%, p=0.68$, Fisher exact test). The slope of the linear trend line after stopping IVlg decreased significantly by -0.310 units per 100 days (95\% confidence interval, -0.611 to $-0.008 ; p=0.04)$.

Conclusions: Recent IVIg exposure is invariably associated with JCV Ab seropositivity. After stopping IVIg, JCV Ab levels tend to decrease with time, and seroreversion to innately Ab-negative status can occur. Neurol Neuroimmunol Neuroinflammation 2014;1:e29; doi: 10.1212/NXI.0000000000000029

\section{GLOSSARY}

$\mathbf{A b}=$ antibody $\mathbf{C I}=$ confidence interval; IRIS = immune reconstitution inflammatory syndrome; IVIg = IV immunoglobulin; $\mathbf{J C V}=$ John Cunningham virus; $\mathbf{m A b}=$ monoclonal antibody; $\mathbf{M S}=$ multiple sclerosis; $\mathbf{n O D}=$ normalized optical density; $\mathrm{PML}=$ progressive multifocal leukoencephalopathy .

John Cunningham virus antibody (JCV Ab) seropositivity is the most important risk factor for progressive multifocal leukoencephalopathy (PML) in patients with multiple sclerosis (MS) treated with natalizumab. ${ }^{1,2}$ Accurate determination of JCV Ab serostatus is critical for clinical decision-making but may not be possible under certain circumstances. For example, plasmapheresis depletes circulating JCV Ab severalfold and may lead to false-negative results in the short-term. ${ }^{3}$ The present article examines an inverse scenario: the impact of therapeutic infusion of IV immunoglobulin (IVIg) on JCV Ab serostatus.

The phenomenon of false-positive serology results following IVIg is well-documented ${ }^{4-6}$ but easily overlooked in practice ${ }^{7-10}$ and clinical trial design. ${ }^{11}$ A case in point is the JCV Antibody Program (STRATIFY-2; NCT01070836; Biogen Idec), which assesses rates of JCV Ab seropositivity in patients with MS who are being treated, or who are considering treatment, with natalizumab. STRATIFY-2 did not exclude patients who were receiving IVIg. ${ }^{12}$

Our 2 centers have enrolled 1,251 patients with MS into STRATIFY-2, of whom 98 patients $(7.8 \%)$ were retroactively identified as having had IVIg exposure during the trial period. The present article examines this subset of IVIg-treated STRATIFY-2 enrollees in order to better understand the effect of IVIg on JCV Ab serostatus and level in serum.

From the NYU Multiple Sclerosis Care Center (I.K., M.O., J.H.), Department of Neurology, NYU School of Medicine, New York, NY; Barnabas Multiple Sclerosis Care Center (I.K., M.Y., J.H.), Department of Medicine, Barnabas Medical Center, Livingston, NJ; Biogen Idec (G.K., M.S.), Cambridge, MA; Department of Epidemiology (E.C.), University of Alabama at Birmingham School of Public Health, Birmingham, AL; and Specialized Clinical Services (K.D.), Park Ridge, NJ.

Go to Neurology.org/nn for full disclosures. Funding information and disclosures deemed relevant by the authors, if any, are provided at the end of the article. The Article Processing Charge was paid by Biogen Idec.

This is an open access article distributed under the terms of the Creative Commons Attribution-Noncommercial No Derivative 3.0 License, which permits downloading and sharing the work provided it is properly cited. The work cannot be changed in any way or used commercially. 
METHODS A total of 1,251 patients with MS were enrolled in STRATIFY-2 at the NYU Multiple Sclerosis Care Center (New York, NY; J.H., principal investigator) and the Barnabas Multiple Sclerosis Care Center (Livingston, NJ; I.K., principal investigator). Medical records of all STRATIFY-2 enrollees from the 2 centers were examined to extract demographic information and to determine which of the participants were exposed to IVIg during the trial period and the time interval from exposure to JCV Ab testing. Pharmacy records were examined to ascertain dates of IVIg administrations. We identified 98 STRATIFY-2 enrollees from the 2 centers who were exposed to IVIg. In our centers, we use IVIg off-label during pregnancy and the postpartum period, ${ }^{13,14}$ as an add-on to the standard disease-modifying therapies, in patients who are unable to tolerate standard therapies, ${ }^{15}$ and in patients with comorbid conditions requiring IVIg, such as chronic inflammatory demyelinating polyneuropathy or immunodeficiency. IVIg brands include Gammagard Liquid (70\% of patients), Privagen (25\%), and Gammaplex and Gamunex-C (5\%).

In addition to STRATIFY-2 trial samples of IVIg-treated enrollees, we included in our analysis STRATIFY JCV and STRATIFY JCV Dx SELECT results obtained commercially as part of routine care (Focus Diagnostics Inc., Cypress, CA). JCV Ab seropositivity was calculated in subgroups of patients: IVIg-naive, JCV Ab-tested within 30 days of IVIg infusion, and JCV Ab-tested more than 30 days after last infusion. The time frame of 30 days was chosen because nearly all of our IVIg-treated patients were on IVIg $0.7 \mathrm{~g} / \mathrm{kg} / \mathrm{month}$

We also calculated the level of JCV Ab in serum, measured in monoclonal JCV antibody ( $\mathrm{mAb}$ ) equivalents, in all samples tested prior to IVIg ("pre-IVIg"), within 30 days of IVIg ("during IVIg"), and more than 30 days after last IVIg exposure ("postIVIg"). The level of polyclonal JCV Ab in patient serum was derived by interpolating the numerical output of the assay for positive samples in normalized optical density (nOD) or index for each sample against a reference curve prepared using a $\mathrm{mAb}$ to $\mathrm{JCV}$. A 5 parameter curve was used to correlate JCV Ab index $\left(R^{2}=1\right)$ or $\mathrm{nOD}\left(R^{2}=0.999\right)$ to JCV mAb level up to an index of about 3 or nOD up to about 1.4 using SoftMax Pro GxP v 5.3 software (Molecular Devices, Sunnyvale, CA). Significance was determined using a nonparametric one-way analysis of variance with Kruskal-Wallis analysis in GraphPad Prism 6.0 software (La Jolla, CA).
For the subset of patients for whom both "during IVIg" and "post-IVIg” JCV Ab levels were available, we estimated mean linear trends while on IVIg and after stopping IVIg with a linear mixed-effects model. The model assumes that "during IVIg" relative concentration and change in relative concentration over time varied randomly among patients according to a multivariate normal distribution with a mean of zero and a covariance matrix that reflected the correlations among repeated observations on the same patient. This model is appropriate when the number of repeated observations is not constant among participants and times of measurement differ from one participant to the other ("unbalanced design"), ${ }^{16}$ as in our study.

Standard protocol approvals, registrations, and patient consents. The study was approved by the institutional review boards of NYU School of Medicine (New York, NY) and Barnabas Medical Center (Livingston, NJ).

RESULTS Numbers of patients and seropositivity rates in the IVIg-naive, "during IVIg," and "post-IVIg" subgroups are shown in the flowchart in figure 1 . The seropositivity rate in the IVIg-naive population (58\%) differed significantly from the seropositivity rate in the "during IVIg" group $(100 \%, p<0.001)$ but not from the "post-IVIg" group $(67 \%, p=0.68$, Fisher exact test).

The relative concentrations of JCV Ab in the first available samples tested prior to IVIg ("pre-IVIg"), within 30 days of IVIg ("during IVIg"), and 30 days after IVIg exposure ("post-IVIg") are shown in figure 2. The relative concentration of the "during IVIg" group (median $2.12 \mu \mathrm{g} / \mathrm{mL}$ ) was significantly greater than the "pre-IVIg" group ( $p=0.0004$; median $0 \mu \mathrm{g} / \mathrm{mL})$ and the "post-IVIg" group $(p<0.0001$; median $0.51 \mu \mathrm{g} /$ $\mathrm{mL})$. The "pre-IVIg" and "post-IVIg" groups were not significantly different from each other $(p=0.72)$.

We identified 20 patients with at least one JCV Ab result within 30 days of IVIg ("during IVIg") and at

Figure 1 Numbers/seropositivity rates of IVIg-naive and IVIg-exposed STRATIFY-2 enrollees

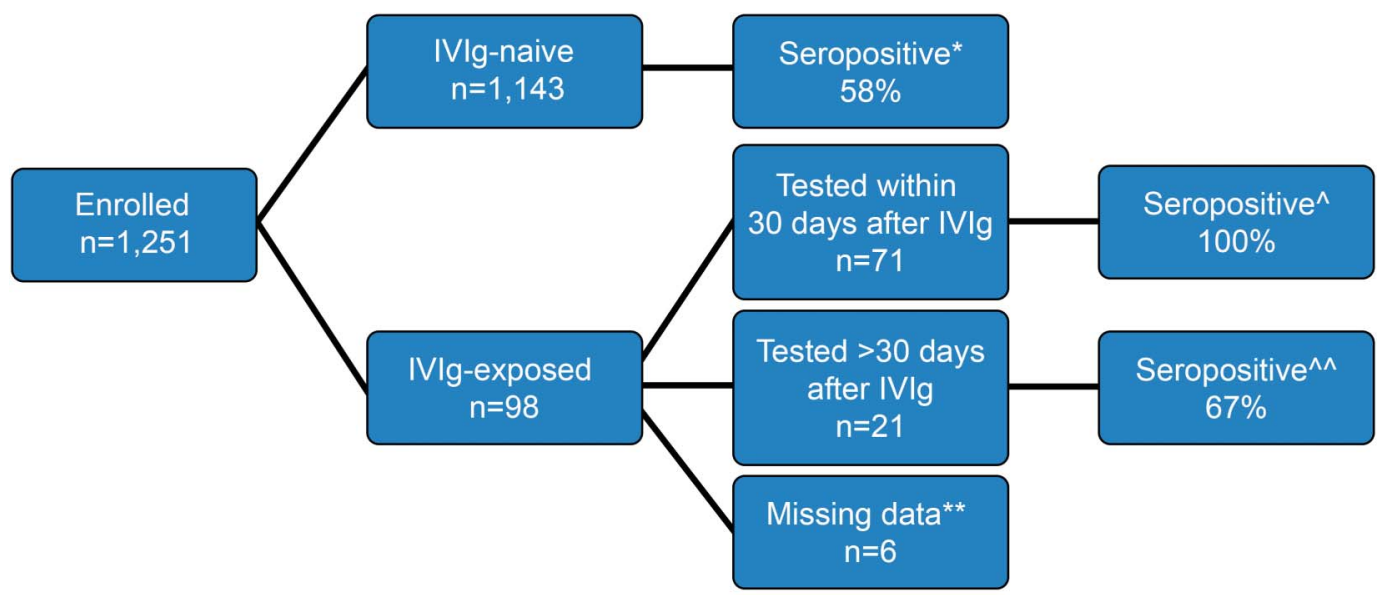

$*=\%$ of enrollment samples, ${ }^{* *}=$ date of IVIg and/or concentration of JCV Ab could not be determined. ${ }^{\wedge}$ indicates that if there was $>1$ sample per patient, we considered the sample tested earlier in time, ${ }^{\wedge}$ indicates that if there was $>1$ sample per patient, we considered the sample tested later. $\mathrm{Ab}=$ antibody; IVIg = IV immunoglobulin; JCV = John Cunningham virus. 
Figure 2 Relative concentration (in mAb equivalents) of JCV Ab in samples tested prior to IVIg ("pre-IVIg"), within 30 days of IVIg ("during IVIg"), and 30 days after IVIg exposure ("post-IVIg")
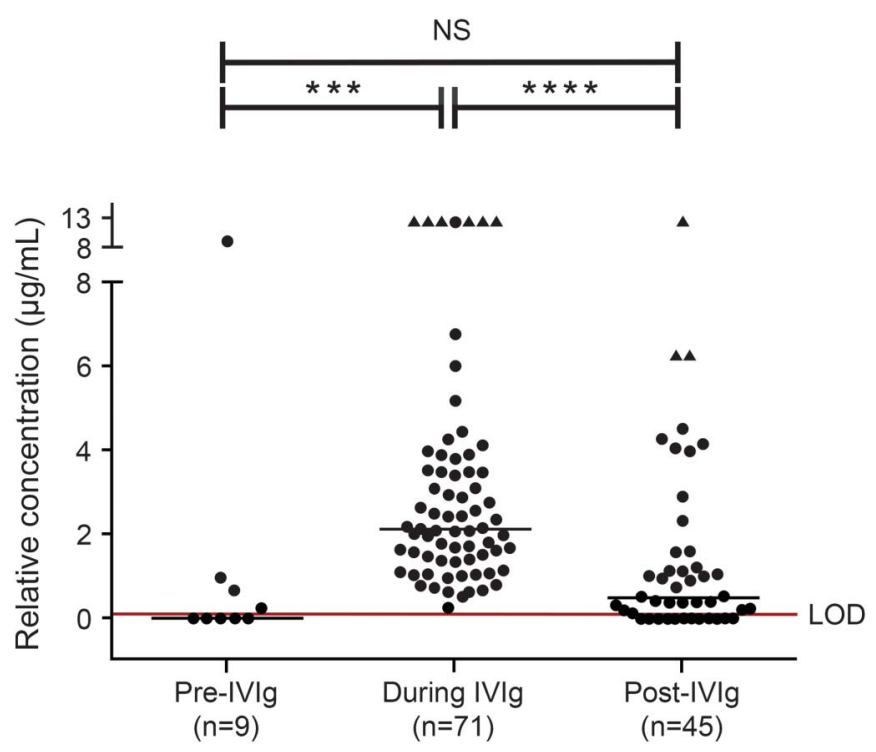

Presence of John Cunningham virus antibody (JCV Ab) in samples collected pre-IVIg ( $n=9)$, within 30 days of IVIg infusion, ("during IVIg," $n=71$ ), and post-IVIg $(n=45)$ was determined using the STRATIFY JCV and STRATIFY JCV Dx SELECT assay. The first available time point for each subject was selected. Six patients are common between the pre-IVIg and during IVlg groups, 24 patients between the during IVIg and post-IVIg groups, and 7 patients between the pre- and post-IVIg groups. The post-IVIg samples range from 31 to 746 days after the last day of IVIg dosing (median 212 days, mean 257 days). The limit of detection (LOD) red line represents the average assay cutpoint translated in monoclonal antibody (mAb) equivalents. The triangle-shaped points represent sample results with concentrations above the limit of relative quantitation possible at the sample dilution tested. Zero relative concentration represents samples that did not have detectable JCV Ab ("seronegative"). The solid line on each graph is the median of the values. The number of samples in the IVIg group is greater than in the groups that are not on IVIg. The statistical test used was one-way analysis of variance with Kruskal-Wallis analysis. IVIg $=\mathrm{IV}$ immunoglobulin. $* * * p=0.0004$, **** $p<0.0001, \mathrm{NS}=$ not significant.

least one JCV Ab result more than 30 days after IVIg was stopped. Using a linear mixed-effects model, the estimated slope of the linear trend line during the treatment phase was not significantly different from that of a flat line (0.066 units per 100 days; $95 \%$ confidence interval $[\mathrm{CI}],-0.188$ to $0.319 ; p=$ 0.61 ; based on 4 patients). The slope of the linear trend line after stopping IVIg decreased significantly by -0.310 units per 100 days $(95 \% \mathrm{CI},-0.611$ to $-0.008 ; p=0.04$; based on 20 patients).

Finally, we present JCV Ab levels in 6 particularly informative patients whose baseline, innate JCV Ab levels were measured prior to initiation of IVIg and who were then retested within 30 days of IVIg exposure and, in 4 patients, $>30$ days after stopping IVIg therapy (figure 3). All 6 subjects showed a marked rise in JCV Ab levels from baseline levels after IVIg was started. JCV Ab levels decreased after IVIg was stopped and 2 out of 3 patients who were seronegative prior to IVIg seroreverted to seronegative status within months of stopping IVIg.
DISCUSSION JCV Ab seropositivity of $58 \%$ in our IVIg-naive group is in excellent agreement with prior reports. ${ }^{17-19}$ In contrast, all 71 patients who received IVIg $0.7 \mathrm{~g} / \mathrm{kg}$ infusion within 30 days of testing were JCV Ab seropositive (100\%). Thus, JCV Ab testing should not be done within 1 month of IVIg exposure.

Our data further suggest that circulating JCV Ab levels decrease after IVIg is discontinued. The seropositivity rate in patients who were tested more than 30 days after IVIg was $68 \%$, which is not significantly different from IVIg-naive patients but is significantly lower than the "during IVIg" group (figure 1). Moreover, mean JCV Ab levels in the IVIg-treated group were significantly higher than in the group that stopped IVIg (figure 2). Of importance, we observed a downward trend in JCV Ab concentration of approximately $1.12 \mathrm{mEq} /$ year in a patient subset for whom we had both "during IVIg" and "post-IVIg" measurements. Taking into account the wide CI, a consequence of small sample size, we would expect that in $95 \%$ of patients, JCV Ab concentration would decrease by $0.03-2.23 \mathrm{mEq}$ over a 1 -year period. This estimate suggests that an innately JCV Ab seronegative patient who experiences modest increases in JCV Ab concentration while on IVIg will likely serorevert to negative status after discontinuation of IVIg. This was indeed observed for 2 out of 3 seronegative patients for whom "pre," "during," and "post" data were available (patients 2 and 6 in figure 3). The only innately seronegative patient who did not revert to seronegative status after discontinuation of IVIg was patient 3 . Although this patient's JCV Ab level dropped precipitously from $1.7 \mathrm{mEq}$ while on IVIg to $0.2 \mathrm{mEq}$ after 51 days off IVIg infusion (patient 3 in figure 3), she was still above the seropositivity cutoff. As JCV Ab levels consistently decrease over time after stopping IVIg, it is probable that even patient 3 would revert to seronegative level after a longer time interval.

Our data have important implications for clinical practice and clinical trial design. Patients with MS may not necessarily be at "high risk" for natalizumabassociated PML based on a positive JCV Ab result if testing was done soon after IVIg (and perhaps other Ig-containing blood products as well). Instead, such patients should be retested for JCV Ab with the expectation that the JCV Ab levels will decrease, on average, by $1.1 \mathrm{mEq}$ over a 12 -month period. Obtaining a more precise estimate of the time interval for retesting IVIgexposed patients would require a prospective study with regularly scheduled JCV Ab testing. ${ }^{20}$ Failure to recognize that IVIg can confound JCV Ab status may result in overestimation of PML risk and consequent denial of a highly effective MS therapy to eligible patients. Although IVIg is not a commonly used MS medication-less than $8 \%$ of our cohort was exposed 

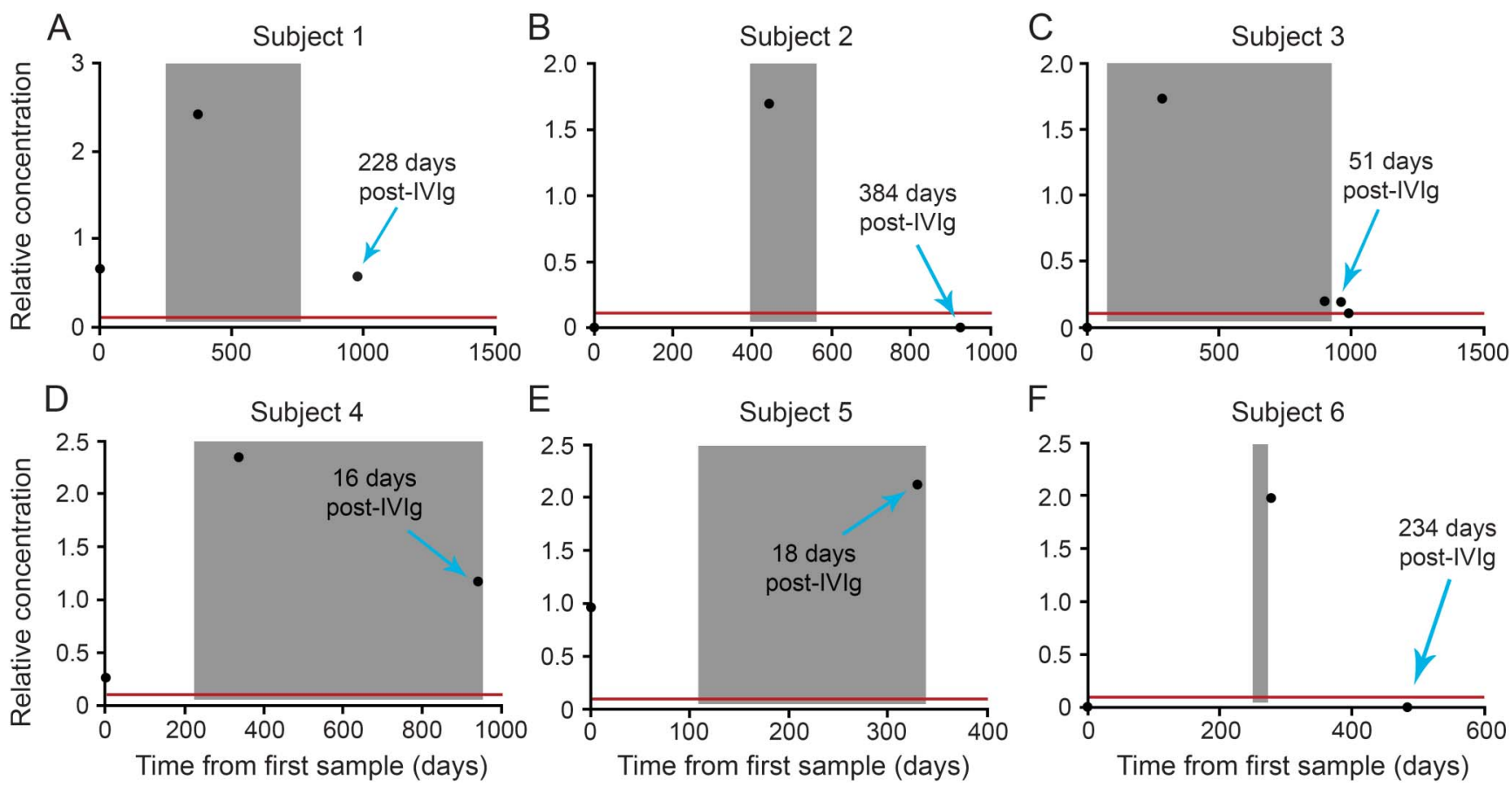

Six patients shown in this figure have had John Cunningham virus antibody (JCV Ab) testing prior to initiation of IV immunoglobulin (IVIg) and within 30 days of IVIg, and patients 1, 2, 3, and 6 also had testing after stopping IVIg therapy (> 30 days after last dose). "Zero" time represents the date of the first sample collected prior to IVIg treatment and is the reference point for other samples and IVIg infusion dates. The shaded area is the time between the first and last IVlg infusion plus 30 days. Zero relative concentration represents samples that did not have detectable anti-JCV Ab. The red line represents the limit of detection of assay translated in monoclonal $(\mathrm{mAb})$ equivalent. All patients showed a significant rise in JCV Ab relative concentration while on IVIg, and most patients showed a significant decrease in relative concentration in mAb equivalents of JCV Ab after stopping IVlg treatment. Two of the 3 patients with appropriate available samples reverted to seronegative several months after stopping IVIg.

to this drug-it is used by some practitioners for prevention of relapses in the peripartum period and for other indications, as described in the Methods section.

We advocate that future trials of serologic markers exclude patients with recent IVIg exposure (or at least require documentation thereof). STRATIFY-2 data should be reexamined to assess the impact of IVIg treatment on JCV $\mathrm{Ab}$ seropositivity assessment. ${ }^{2}$ Lastly, significant elevation of JCV Ab levels in serum following IVIg infusion raises a possibility that IVIg may have a role in the management of PML/immune reconstitution inflammatory syndrome (IRIS). A study of IVIg, a safe and well-tolerated therapy, on PML-IRIS should test this hypothesis.

\section{AUTHOR CONTRIBUTIONS}

Ilya Kister: study concept and design, analysis and interpretation of data, drafting of the manuscript, critical revision of the manuscript for important intellectual content. Geoffrey Kuesters: collection, analysis, and interpretation of data; drafting of the manuscript; critical revision of the manuscript for important intellectual content. Eric Chamot: analysis and interpretation of data, critical revision of the manuscript for important intellectual content. Mirza Omari: collection and analysis of data, critical revision of the manuscript for important intellectual content. Kim Dontas: collection and analysis of data, critical revision of the manuscript for important intellectual content. Mary Yarussi: collection and analysis of data, critical revision of the manuscript for important intellectual content. Meena Subramanyam: analysis and interpretation of data, drafting of the manuscript, critical revision of the manuscript for important intellectual content. Joseph Herbert: study concept and design, analysis and interpretation of data, drafting of the manuscript, critical revision of the manuscript for important intellectual content, study supervision.

\section{ACKNOWLEDGMENT}

This work was supported by an investigator-initiated research grant from Biogen Idec. The authors are grateful to the pharmacists at Bioscrips and AOM for verifying information on IVIg usage in our patients and to Dr. Ilir Topalli for fruitful discussions.

\section{STUDY FUNDING}

The study was supported in part by an investigator-initiated grant from Biogen Idec.

\section{DISCLOSURE}

I. Kister received research support from the National Multiple Sclerosis Society (NMSS), Jackson-Guthy Charitable Foundation, EMD-Serono/ Pfizer, Inc., Biogen Idec, Serono, and Novartis and received consulting fees from Biogen Idec. G. Kuesters is an employee of and holds stock with Biogen Idec. E. Chamot has served as a member of the Multiple Sclerosis Outcome Assessments Consortium (MSOAC) of the National Multiple Sclerosis Society and as a member of the International MSFC Task Force of the National Multiple Sclerosis Society and has received research support from the NIH, NMSS, UAB Comprehensive Cancer Center-AIDS Research Center, and Consortium of Multiple Sclerosis Center. M. Omari, K. Dontas, and M. Yarussi report no disclosures. M. Subramanyam holds a patent for use of Anti-JCV antibody index for PML risk stratification, Method of assessing risk of PML ELISA to determine clinically meaningful anti-drug antibodies and is an employee of and holds stock in Biogen Idec. J. Herbert has received funding from Biogen and Genzyme and has received research support from Novartis, Biogen Idec, Acorda, Roche, Genentech, 
Teva, Genzyme, and St. Barnabas Hospital. Go to Neurology.org/nn for full disclosures.

Received April 30, 2014. Accepted in final form August 13, 2014.

\section{REFERENCES}

1. Gorelik L, Lerner M, Bixler S, et al. Anti-JC virus antibodies: implications for PML risk stratification. Ann Neurol 2010;68:295-303.

2. Bloomgren G, Richman S, Hotermans C, et al. Risk of natalizumab-associated progressive multifocal leukoencephalopathy. N Engl J Med 2012;366:1870-1880.

3. Subramanyam M, Plavina $T$, Khatri BO, Fox RJ, Goelz SE. The effect of plasma exchange on serum antiJC virus antibodies. Mult Scler 2013;19:912-919. doi: 10. $1177 / 1352458512467502$.

4. Lichtiger B, Rogge K. Spurious serologic test results in patients receiving infusions of intravenous immune gammaglobulin. Arch Pathol Lab Med 1991;115:467-469.

5. Horst HA, Schmitz N, Glinike C, Löffler H, Laufs R. Seroconversion for hepatitis $\mathrm{C}$ virus antibody in bone marrow recipients treated with immune globulin. $\mathrm{N}$ Engl J Med 1991;325:132-133.

6. Prohaska W, Wolff C, Schlüter K, Köster-Eiserfunke W, Körner MM, Kleesiek K. Immunoglobulin preparations from hepatitis $\mathrm{C}$ antibody-positive plasma donors: influence on diagnosis and risk of infection in heart transplant recipients. Clin Investig 1992;70:573-578.

7. Lopez-Jimenez J, Villalon L, Mateos ML, Odriozola J. Hepatitis $\mathrm{C}$ virus antibody seroconversion in bone marrow transplant recipients treated with immune globulin: the impact of the problem. Blood 1994;84:665-666.

8. Bélanger SS, Fish D, Kim J, Cohen S. False-positive human T-lymphotropic virus serology after intravenous immunoglobulin transfusion. CMAJ 2012;184:1709_ 1712. doi: 10.1503/cmaj.120019.

9. Hunter M, Kiernan MC, Post J. Human T-lymphotropic virus seroconversion associated with pooled human intravenous immunoglobulin therapy. Intern Med J 2012;42: 729-730. doi: 10.1111/j.1445-5994.2012.02803.x.

10. Rossi KQ, Nickel JR, Wissel ME, et al. Passively acquired treponemal antibody from intravenous immunoglobulin therapy in a pregnant patient. Arch Pathol Lab Med 2002;126:1237-1238.

11. Arnold DM, Crowther MA, Meyer RM, et al. Misleading hepatitis B test results due to intravenous immunoglobulin administration: implications for a clinical trial of rituximab in immune thrombocytopenia. Transfusion 2010;50: $2577-2581$.

12. JCV Antibody Program in Patients With Relapsing Multiple Sclerosis Receiving or Considering Treatment With Tysabri: STRATIFY-2. Available at: http://clinicaltrials. gov/ct2/show/NCT01070836. Accessed January 18, 2014.

13. Achiron A, Kishner I, Dolev M, et al. Effect of intravenous immunoglobulin treatment on pregnancy and postpartumrelated relapses in multiple sclerosis. J Neurol 2004;251: 1133-1137.

14. Haas J, Hommes OR. A dose comparison study of IVIG in postpartum relapsing-remitting multiple sclerosis. Mult Scler 2007;13:900-908.

15. Elovaara I, Apostolski S, van Doorn P, et al. EFNS guidelines for the use of intravenous immunoglobulin in treatment of neurological diseases: EFNS task force on the use of intravenous immunoglobulin in treatment of neurological diseases. Eur J Neurol 2008;15:893-908.

16. Fitzmaurice GM, Laird NM, Ware JH. Applied Longitudinal Analysis, 2nd ed. Hoboken, NJ: Wiley; 2011.

17. Bozic C, Richman S, Plavina T, et al. Anti-John Cunnigham virus antibody prevalence in multiple sclerosis patients: baseline results of STRATIFY-1. Ann Neurol 2011;70:742-750.

18. Trampe AK, Hemmelmann C, Stroet A, et al. Anti-JC virus antibodies in a large German natalizumab-treated multiple sclerosis cohort. Neurology 2012;78:1736-1742.

19. Outteryck O, Ongagna JC, Duhamel A, et al. Anti-JCV antibody prevalence in a French cohort of MS patients under natalizumab therapy. J Neurol 2012;259:22932298.

20. Rand KH, Houck H, Ganju A, Babington RG, Elfenbein GJ. Pharmacokinetics of cytomegalovirus specific IgG antibody following intravenous immunoglobulin in bone marrow transplant patients. Bone Marrow Transpl 1989;4:679-683. 


\section{Neurology \\ Neuroimmunology \& Neuroinflammation}

IV immunoglobulin confounds JC virus antibody serostatus determination

Ilya Kister, Geoffrey Kuesters, Eric Chamot, et al.

Neurol Neuroimmunol Neuroinflamm 2014;1;

DOI 10.1212/NXI.0000000000000029

This information is current as of September 18, 2014

\section{Updated Information \& Services}

References

Subspecialty Collections

Permissions \& Licensing

\section{Reprints}

including high resolution figures, can be found at:

http://nn.neurology.org/content/1/3/e29.full.html

This article cites 18 articles, 2 of which you can access for free at: http://nn.neurology.org/content/1/3/e29.full.html\#\#ref-list-1

This article, along with others on similar topics, appears in the following collection(s):

All Immunology

http://nn.neurology.org//cgi/collection/all_immunology

Multiple sclerosis

http://nn.neurology.org//cgi/collection/multiple_sclerosis

Information about reproducing this article in parts (figures,tables) or in its entirety can be found online at:

http://nn.neurology.org/misc/about.xhtml\#permissions

Information about ordering reprints can be found online:

http://nn.neurology.org/misc/addir.xhtml\#reprintsus

Neurol Neuroimmunol Neuroinflamm is an official journal of the American Academy of Neurology.

Published since April 2014, it is an open-access, online-only, continuous publication journal. Copyright $(\subseteq$ 2014 American Academy of Neurology. All rights reserved. Online ISSN: 2332-7812.

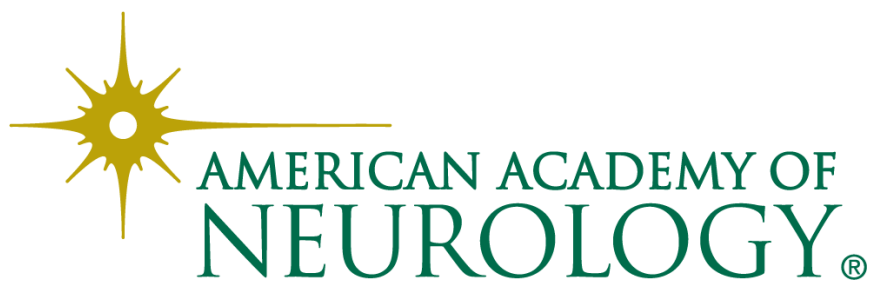

